\title{
Thar She Blows: Can Bubbles Be Rekindled with Experienced Subjects?
}

\author{
Reshmaan N. Hussam \\ Massachusetts Institute of Technology \\ David Porter and Vernon L. Smith \\ Economic Science Institute \\ Chapman University
}

\begin{abstract}
We report 28 new experiment sessions consisting of up to three experience levels to examine the robustness of learning and 'error' elimination by participants in a laboratory asset market and its effect on price bubbles. Our answer to the title question is: "yes." We impose a large increase in liquidity and dividend uncertainty to shock the environment of experienced subjects who have converged to equilibrium, and this treatment rekindles a bubble. However, in replications of that challenging environment across three experience levels we discover that the environment yields a rare residual tendency to bubble even in the third experience session. Therefore, a caveat must be placed on the effect of twice experienced subjects in asset markets: in order for price bubbles to be extinguished, the environment in which the participants engage in exchange must be stationary and bounded by a range of parameters. Experience, including possible 'error' elimination, is not robust to major new environment changes in determining the characteristics of a price bubble.
\end{abstract}


Conducting laboratory asset market experiments has been standard fare since it was first found by Smith, Suchanek and Williams [1988; hereafter SSW] that price bubbles could be reproduced in settings where information on fundamental value was repeatedly made public. ${ }^{1}$ Many laboratory replications of this phenomenon have been conducted under a variety of treatments. Researchers have found that the occurrence of price bubbles persists with treatments such as transactions or brokerage fees on trading, capital gains taxes, short-selling ${ }^{2}$, identical portfolios across all participants, or the use of subjects drawn from subpopulations of corporate managers, independent small business persons or professional stock traders (see King et al. [1993] and Lei et al. [2001]). These latter treatments have converted the oft' heard claim that 'it's only undergraduates' to 'it seems to be everybody' in this class of environments. ${ }^{3}$ More pronounced price bubbles have also been found via the allowance of margin buying and/or increased levels of liquidity in

1 We refer to "information" on value, not "knowledge" of value as in SSW because there is an important distinction between the two terms that is particularly relevant in experimental science: the distinction between "knowledge that," which is a good synonym for "information," and "knowledge how;" the latter involves also all of the individual's experience, past information and acquired tacit knowledge of how to function, plus discovering all you need to learn to function effectively in a new environment with new information. Thus, what the bubble experiments show is that over time (after two sessions of experience) people come to have common knowledge of fundamental value in the sense that such information has become integrated into the group's tacitly acquired knowledge of how to function effectively with that information. These considerations are central to the conceptual distinction between constructivist and ecological rationality discussed in Smith (2003).

${ }^{2}$ Ackert, Charupat, Church, and Deaves (2006) alter the institutional design of their short sale feature from that of King et al. (1993) to better reflect short sale practice in real markets. They find that the allowance of such short sales drives prices towards and often below fundamental value, and conclude that short selling helps moderate bubbles in experimental asset markets. However, Haruvy and Noussair (2006), finding similar results of often "negative" bubbles in the presence of short sales, argue that the allowance of short selling tends to reduce prices absolutely rather than inducing any rational expectations in subjects which move them to trade at fundamental value.

3 Except see McCabe and Smith (2000) in which inexperienced graduate students in economics trade at fundamental dividend value throughout the horizon. But well over half the subjects from the same samples decline to choose dominant strategies in two-person extensive form trust games. Hence, there should be no presumption that individual "rationality" as theoretically defined is a generic characteristic of more sophisticated subjects. 
the form of cash endowments (see King et al. [1993], Caginalp et al. [1998]). It should be emphasized, however, that in all cases asset prices in these markets converge across experience levels to the intrinsic rational fundamental value of the asset. ${ }^{4}$ Hence, equilibrium theory, which says nothing about the process and speed of equilibrium convergence, is not contradicted by this evidence. What has been falsified is the hypothesis that all information relevant to share value is immediately incorporated into its trading price by consciously rational calculation and action by traders based on the information they are provided before trading begins.

There are several treatments that seem to lessen the effects of a bubble such as introducing a futures market or constraining opening market prices (at the beginning of the asset's life) to occur near fundamental value (see Porter and Smith [1995], Caginalp et al. [2000], Noussair and Tucker [2006]). But the only condition that has reliably eliminated price bubbles in these environments and yielded convergence toward fundamental value is increased experience in the same environment. In particular, SSW and subsequent replications found that common group experience, that is, the same cohort of traders, who can see they are the same, causes trading to thin out and contract prices to converge toward fundamental value by the third replication of the market. For example, Figure 2 shows a common time series pattern over a 15-period horizon for the same cohort of traders in three trading sessions, from inexperienced to twice-experienced.

\footnotetext{
$4 \quad$ However, in Dufwenberg, Lindqvist and Moore [2005], their environment generates price bubbles that do not converge but grow throughout the time horizon. In Figure 1 we provide a graph of the average contract prices in their experiments for different levels of experience. These results differ markedly from the typical asset market experiment price pattern of a bubble and subsequent crash to fundamental value or the twice-experienced result of prices at fundamental value over most of the horizon. This suggests that the character of an asset market price bubble is directly related to the environment's parameters.
} 
Note that both the deviation of mean contract prices from fundamental value and the trading volume decline with experience. Moreover, Porter and Smith [1995] found that there is an interaction between experience and the variance of the dividend distribution. For example, when the per-period dividend is certain, it takes only once-experienced subjects to eliminate a price bubble.

Dufwenberg, Lindqvist and Moore [2005] (hereafter referred to as DLM) report experiments in single sessions in which a cohort of six subjects participate in a sequence of 4 ten-period standard asset markets with identical initial parameters. In their fourth 10period market, either 2 or 4 of the six experienced subjects are randomly selected and replaced with inexperienced subjects. They find that with these levels of inexperienced subjects, trading occurs at levels similar to those of twice experienced cohorts. However, their results are not directly comparable with those reported in SSW because of key differences in experimental design: SSW's experiments used more traders (9-12) in longer (15 period) horizons with a dividend distribution with four potential outcomes and subjects who returned in separate experienced sessions. ${ }^{5}$

But the results reported by DLM establish that there does exist an environment in which identical cohort interaction may not be necessary for experience to achieve its diminishing effect on a bubble. In a given asset market environment, repetition (experience) for as little as one-third of the six subjects yielded trade similar to that of

$5 \quad$ DLM point out that both SSW and Petersen [1993] conducted some mixed experience asset market experiments with varied results. In addition, King et al. [1993] used insiders and found that the bubbles remained and sometimes did not crash if short-selling was allowed, however, Haruvy and Noussair [2006] find that short selling can moderate bubbles. 
experienced cohorts. Because of differences in the economic environment and procedures this is not inconsistent with the small sample of experiments reported in SSW also showing that bubbles were dampened in groups composed of a larger proportion of experienced relative to inexperienced subjects.

In addition to the negative correlation between bubbles and experience, 'errors ${ }^{6}$ in decision making by subjects have been observed; i.e., it has been posited that 'confusion,' or mistaken understanding and analysis of the asset trading environment, leads to 'irrationality' at the individual level that is associated with bubbles (Lei et al. [2001]). Specifically, Lei et al. changed the trading rules so that buyers are not permitted to resell shares purchased and can only buy or hold against dividend realizations while sellers can only sell against cash or hold for dividends. This clever design provides a test of the interpretive hypothesis that buyers (sellers) buy strategically for resale (sell for repurchase) because it is hypothesized that they expect prices to rise (fall). In this theoretical construct of the trader there is a disequilibrium phase in which bubbles arise because buyer expectations of capital gains predominates the counter sentiment of sellers, and via backward induction, buying for subsequent resale predominates over selling for subsequent repurchase. Lei et al. falsify this expectations-backward-induction model of the trader, by demonstrating that their trading rule constraints on buying for resale and selling for repurchase do not prevent bubbles; rather, price bubbles do survive, making it clear that our standard analysis of expectations and decision-making does not apply to the subjects.

$6 \quad$ By error we mean trade occurring at prices that deviate significantly from fundamental value. 
To further clarify this interpretation, notice that this finding does not tell us anything about how traders actually think, what they do, how they adapt to their environment, or why they eventually get it right through experience; rather these findings inform us about what these traders do not do. In particular, they do not think about the problem the way we do as economists, namely to backward induct from future anticipated prices and maximize expected value before taking action. Hence their 'error' is measured relative to our way of thinking, a way of thinking that correctly predicts their behavior after they become 'sufficiently' experienced, but which can predict neither how much experience is needed nor their thinking process. What are missing in economic modeling are alternative theories of agent adaptation besides the backward-induction-optimal-decision approach or deviants thereof. Bubbles are the funny and unpredictable phenomena that happens on the way to the 'rational' predicted equilibrium if the environment is held constant long enough. But we lack good characterizations or models of agent thought/decision processes that explain what they do, how they adapt, and why they eventually converge. We do know that in asset trading (and many other) environments experience reduces error, where 'error' is defined as the discrepancy between predicted and observed behavior. For this reason we prefer to use the word 'error' rather than 'confusion' in referring to such discrepancies.

In this paper, we will examine the robustness of market experience. In particular, we ask the question: Can a price bubble be rekindled with twice-experienced subjects? If twiceexperienced subjects can be induced to trade at price levels and volumes similar to less 
experienced subjects, then the robustness of being twice experienced in eliminating bubbles is challenged. Our basic approach in the design used here is to simply retool it using earlier results showing that bubbles are exacerbated by yield (dividend) uncertainty and by available liquidity, and apply such treatments to twice-experienced subjects. If one thinks of great stock market booms as driven by waves of new technology, such environments introduce new sources of unpredictable yield uncertainty, and parallel with this development we see much new liquidity attracted into equity investment. Thus, we insert these two conditions to measure their ability to rekindle bubbles among twiceexperienced subjects. We also draw on widespread experimental evidence that 'learning' (qua adaptation) is context (environment) dependent and does not effectively transfer quickly to altered environments. This in itself also suggests that subjects do not think about their task, and generalize from it, the way we do using economic reasoning.

\section{Experimental Design}

Our experiment environment uses the canonical asset market form in which a security with a finite life of 15 periods is traded. ${ }^{7}$ The asset pays a random dividend drawn from a fixed distribution each period. In our baseline experiments, the dividend distribution was uniform over the four potential outcomes $\{0,8,28,60\}$ in cents. Thus, the expected dividend payout each period was 24 cents. Over a 15-period horizon, the asset should begin at a fundamental value of 360 and decline by 24 each period. In addition to this asset value structure, each subject was endowed with an initial portfolio of shares and

$7 \quad$ We used a conventional uniform price sealed bid-offer call market institution to reallocate shares from sellers to buyers each period at one market clearing price. We are comfortable with studies showing that call markets exhibit bubble properties equivalent to those of the continuous double auction in comparisons using the same asset market environment (see Van Boening et al. [1993], Caginalp et al. [2000] and Haruvy et al. [2007]). 
cash. We use three portfolio types that we spread evenly across subjects. Table 1 lists these three portfolio types. Thus, the average portfolio consisted of 4 shares and 720 in cash. ${ }^{8}$ We call this environment the baseline.

Within the baseline environment, we developed specific protocols of subject experience following SSW. We recruited subject cohorts from the undergraduate population at George Mason University who participated in the baseline market and then each group was brought back for a second experiment. We refer to these two sessions as the baseline sessions. We cycled five cohorts through two sessions each, so that each cohort had one session as inexperienced traders and the second as once-experienced traders. We then took this pool of 70 once-experienced subjects and mixed them for a third session. In this way we had a set of once-experienced subjects in cohorts in which the subjects could see that the composition of their group was not identical to the groups they had been in before. In addition to mixing the subjects, we changed two other environment variables that have been shown to increase the severity of a bubble. Specifically, we increased the variance of the dividend distribution and increased liquidity in the market by lowering the initial number of shares and increasing the amount of cash. We call this the rekindle treatment. Dividends were now drawn with equal probabilities for each of five potential outcomes $\{0,1,8,28,98\}$ so that the one-period expected dividend value was 27 . The change in the initial portfolio of cash and shares was set so that the cash positions were

\footnotetext{
$8 \quad$ The expected baseline portfolio value is initial cash + (initial shares) $\mathrm{x}$ (per period expected share dividend) $x(15$ periods). For example, $\$ 7.20+(4) \times(\$ .24) \times(15)=\$ 21.60$.
} 
doubled and the outstanding stock was cut in half. Table 2 shows the portfolio values for the rekindle treatment. ${ }^{9}$

To avoid any misunderstanding we want to emphasize that our rekindle environment is not one that is tweaked only moderately or slightly. We double the cash endowments and halve the share endowments in rekindle relative to baseline. We also (slightly) increase the per-period expected dividend, but increase substantially its variance, from 715 to 1966. Hence, we shock the environment using twice previously experienced subjects from the baseline environment to see if we can rekindle a bubble in spite of that experience. We have not supposed that re-ignition with highly experienced subjects would be easy. The experiments are deliberately designed to push the edge of what has been the conventional learning — an important unique function of lab experiments. We think the cited earlier studies have discovered how to 'reliably' get equilibrium behavior. The question herein is how robust is that finding: we are asking if being twice previously experienced will dampen, arrest, or modify trading away from dividend value under the shock.

In advance of doing the experiments we (and no one) could say what kinds of changes it would take to empirically identify conditions under which subjects behaved as if they were in a different game. For making these comparisons we draw on a data base of experiments (see Table 3) we conducted over the years with a standard 15-period market

\footnotetext{
9 The large changes are not in expected portfolio value but in the hypothesized neutral mix of cash
} and share endowments and the range/variance of the (roughly mean preserving) distribution of dividends. 
in which experienced sessions are with same subject cohorts in a constant environment, and therefore are in a position to provide an answer to this question.

From the 5 baseline sessions with 70 subjects, we conducted 3 rekindle sessions with a total of 33 subjects. We also conducted two independent series of replication experiments, each with three sessions. These were identical to the baseline experiments except for the presence of a third session, in which twice-experienced subjects returned for a third time with the same cohort of traders and same environment. This was done in order to replicate the results in SSW with our subject pool. This procedure is intended to control for any inference error due to the possibility that our results in the rekindle experiments reflect sampling peculiarities in our subject pool. Figure 3 plots the results of the replication experiments which are consistent with previous experimental results under this environment.

Finally, in addition to the baseline, rekindle, and replication sessions, we recruited 45 more subjects to participate in a set of replication experiments with rekindle parameters, termed new replication. Specifically, we set up three experiments with three sessions each. Each experiment had a cohort of fifteen subjects who, as in SSW, went through the three sessions (experiences) together. The parameters for all sessions were static and equivalent to the rekindle parameters. This design allowed us to observe the bubble pattern across all three experience levels holding the rekindle environment constant 
throughout. $^{10}$

\section{Experimental Results}

Figure 4 shows the three rekindle experiment price time series along with the two twiceexperienced cohorts using our baseline parameters. It is clear from this chart that there is a difference between the rekindle treatment and the twice-experienced subjects in the replication treatment (with baseline parameters). Figure 5 plots the three new replication experiments along with the two twice-experienced baseline cohorts, establishing that the rekindle parameters continue to induce bubble behavior into the twice-experienced sessions. Hence, to be more precise and complete in taking account of sampling variability, we analyze the experiments by examining four primary bubble characteristics defined by each session:

1. Amplitude: this variable measures the trough-to-peak change in market asset value relative to fundamental value. Formally, this is measured as $\mathrm{A}=\operatorname{Max}\left\{\frac{P_{t}-f_{t}}{E}: t=1, \ldots, 15\right\}-\operatorname{Min}\left\{\frac{P_{t}-f_{t}}{E}: t=1, \ldots, 15\right\}$ where $P_{t}$ is the market price in period $\mathrm{t}, f_{t}$ is the fundamental value of the asset in period $\mathrm{t}$, and $E$ is the expected dividend value over the life of the asset.

2. Duration: this variable measures the length, in periods, in which there is an observed increase in market prices relative to fundamental value. Formally,

$10 \quad$ We are grateful to one of our referees for urging us to conduct these additional comparison experiments the results of which modified and extended our initial conclusions based only on the rekindle treatment. 
duration is defined as $\mathrm{D}=\operatorname{Max}\left\{m: P_{t}-f_{t}<P_{t+1}-f_{t+1}<\ldots .<P_{t+m}-f_{t+m}\right\}$. For example, in Figure 4 experiment rekindle 2 has a duration of 4 periods.

3. Turnover: this variable measures the trading activity in the market. Formally, this is measured as $\mathrm{T}=\frac{\sum_{t} V_{t}}{S}$, where $\mathrm{V}$ is the volume of trade in period $\mathrm{t}$ and $\mathrm{S}$ is the total outstanding stock in the experiment.

4. Market Value Amplitude: this variable measures the normalized market value of trade. That is, we weight period amplitude by the volume of trade. Formally, $\mathrm{M}=\operatorname{Max}\left\{\frac{P_{t}-f_{t}}{E} V_{t}: t=1, \ldots . .15\right\}$.

A priori there might be good reason to be skeptical of this empirical analysis: there exists no general way to represent a time path of observations with a scalar variable. We use four scalars, but these time paths have important empirical characteristics that belie generality. Empirically bubble paths are extinguished over time and show regularities in their pattern: the normalized price amplitude, which tends to be single-peaked, declines with experience; the corresponding turning point periods tend to be single valued and to decline with experience; turnover on average declines monotonically with experience. Although individual measures of each element in this 4-tuple are subject to high sampling variability, this quality in their regularity tends to be preserved. This is particularly captured in Figure 2, which, however, does not illustrate the sampling variability. The relatively large sample of experiments in the data base we report in Table 3 is important in reducing the standard error of this sampling variability. Hence, much of the information content of a bubble is captured in these four scalar reductions. Moreover, 
this regularity is part of the theoretical challenge that needs to be explained by the appropriate dynamic financial model.

For the regression analysis below, in addition to the 28 experiment sessions described above, we use the results from 53 previous 15-period asset market experiments with inexperienced, once-experienced and twice-experienced subjects in the baseline environment (the data come from SSW, Caginalp et al. [2000], King et al. [1993], Van Boening et al. [1993] and Caginalp et al. [2001]). Across all the data, if experience is robust we should find that the characteristics of the rekindle treatment should be equivalent to the twice-experienced treatments and bubbling should diminish with experience in the new replication treatments. Also the rekindle and twice experienced new replication should be comparable. In Table 5 we provide the appropriate pairwise comparisons.

We estimate the following seemingly unrelated regressions (SUR):

Amplitude $_{i}=$ Intercept $+\beta_{1 \mathrm{~A}}$ Once-experienced $_{\mathrm{i}}+\beta_{2 \mathrm{~A}}$ Twice-experienced $_{\mathrm{i}}+\beta_{3 \mathrm{~A}}$ Rekindle $_{\mathrm{i}}+$ $\beta_{4 \mathrm{~A}}$ Inexperience-NewRep $_{\mathrm{i}}+\beta_{5 \mathrm{~A}}$ Once-exper.-NewRep ${ }_{\mathrm{i}}+\beta_{6 \mathrm{~A}}$ Twice-exper.-NewRep $_{\mathrm{i}}+$ $\beta_{7 \mathrm{~A}}$ Inexperienced-Replication $_{\mathrm{i}}+\beta_{8 \mathrm{~A}}$ Once-exper.-Replication $_{\mathrm{i}}$

Duration $_{i}=$ Intercept $+\beta_{1 \mathrm{D}}$ Once-experienced ${ }_{\mathrm{i}}+\beta_{2 \mathrm{D}}$ Twice-experienced $_{\mathrm{i}}+\beta_{3 \mathrm{D}}$ Rekindle $_{\mathrm{i}}+$ $\beta_{4 \mathrm{D}}$ Inexperience-NewRep $_{\mathrm{i}}+\beta_{5 \mathrm{D}}$ Once-exper.-NewRep ${ }_{\mathrm{i}}+\beta_{6 \mathrm{D}}$ Twice-exper.-NewRep ${ }_{\mathrm{i}}+$ $\beta_{7 \mathrm{D}}$ Inexperienced-Replication $_{\mathrm{i}}+\beta_{8 \mathrm{D}}$ Once-exper.-Replication $_{\mathrm{i}}$

Turnover $_{\mathrm{i}}=$ Intercept $+\beta_{1 \mathrm{~T}}$ Once-experienced ${ }_{\mathrm{i}}+\beta_{2 \mathrm{~T}}$ Twice-experienced $_{\mathrm{i}}+\beta_{3 \mathrm{~T}}$ Rekindle $_{\mathrm{i}}+$ $\beta_{4 \mathrm{~T}}$ Inexperience-NewRep $_{\mathrm{i}}+\beta_{5 \mathrm{~T}}$ Once-exper.-NewRep ${ }_{\mathrm{i}}+\beta_{6 \mathrm{~T}}$ Twice-exper.-NewRep $_{\mathrm{i}}+$ $\beta_{7 \mathrm{~T}}$ Inexperienced-Replication $_{\mathrm{i}}+\beta_{8 \mathrm{~T}}$ Once-exper.-Replication $_{\mathrm{i}}$ 


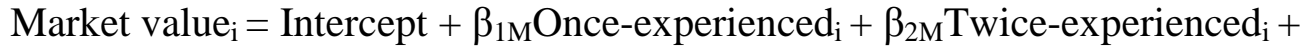
$\beta_{3 M}$ Rekindle $_{i}+\beta_{4 M}$ Inexperience-NewRep ${ }_{i}+\beta_{5 M}$ Once-exper.-NewRep ${ }_{i}+\beta_{6 M}$ Twice-exper.NewRep $_{i}+\beta_{7 M}$ Inexperienced-Replication $_{i}+\beta_{8 \mathrm{M}}$ Once-exper.-Replication $_{\mathrm{i}}$

Where $\mathrm{i}$ denotes the session, the independent variables are $\{0,1\}$ dummy variables denoting the treatment, and $\beta_{\mathrm{jk}}$ is a coefficient denoting treatment $\mathrm{j}$ and measurement $\mathrm{k}$. The results of SUR estimates are provided in Table 4. The estimates from this table allow us to conclude:

Result 1: Experience reduces the amplitude of a bubble significantly. However, experience with the rekindle treatment results in a bubble amplitude that is no different than the amplitude of inexperienced subjects. Moreover, relative to twice-experienced cohorts, rekindle has a greater amplitude.

In addition to the t-statistics derived from the SUR regressions, Table 5 provides the results of the Exact Wilcoxon Rank sum test for the null hypothesis that the rekindle amplitude is the same as inexperienced versus the two-sided alternative with $W=49, n=3$, $\mathrm{m}=34$, $\mathrm{p}$-value $=0.6532$; for rekindle versus twice-experienced it is $\mathrm{W}=30, \mathrm{n}=3, \mathrm{~m}=8$, $\mathrm{p}$ value $=0.0121$. This result shows that an environment-specific type of experience is required in this data set to eliminate bubbles. In the static baseline environment, we can rely on experience to eliminate a bubble. However, once the underlying market parameters of liquidity, dividend uncertainty and unfamiliar faces have been altered to the rekindle values, experience is not a sufficient condition to eliminate the amplitude of a price bubble. 
Result 2: Experience significantly reduces the duration of a bubble. The experience in the rekindle treatment also shows a reduced duration. Thus, while experience with rekindling does not have an effect on the amplitude of the bubble it reduces its duration. The reduction, however, is not as large as that in the twice-experienced baseline environment.

From Table 5, the Exact Wilcoxon Rank sum test for the null hypothesis that the rekindle duration is the same as inexperienced versus the two-sided alternative $\mathrm{W}=11, \mathrm{n}=3, \mathrm{~m}=34$, $\mathrm{p}$-value $=.0041$; for rekindle versus twice-experienced it is $\mathrm{W}=27, \mathrm{n}=3, \mathrm{~m}=8$, $\mathrm{p}$ value $=.0848$. Result 2 in comparison with result 1 shows that there is a residual effect of experience on the market. While experience alone does not reduce the size of a bubble, it does reduce its duration. Participants seem to be tacitly aware that there will be a crash and consequently exit from the market (sell) earlier, causing the crash to start at an earlier period.

Result 3: Experience significantly reduces turnover. This does not carry over to the rekindle treatment. Turnover in rekindle is not significantly different than the turnover with inexperienced subjects.

The Exact Wilcoxon Rank sum test for the null hypothesis that the rekindle turnover is the same as inexperienced versus the two-sided alternative is $\mathrm{W}=31, \mathrm{n}=3, \mathrm{~m}=34, \mathrm{p}$ value $=0.1647$; for rekindle versus twice-experienced it is $\mathrm{W}=27, \mathrm{n}=3, \mathrm{~m}=8, \mathrm{p}$-value $=$ 0.0848. Result 3 is perhaps not surprising given the high level of cash-to-shares in the 
rekindle treatment. If investors are cash rich, they look for spending opportunities during the periods of disequilibrium.

Result 4: Experience significantly reduces market value amplitude. This carries over to the rekindle treatment. However, it is not reduced to the level found with twice experienced subjects in the baseline.

The Exact Wilcoxon Rank sum test for the null hypothesis that the Rekindle market value amplitude is the same as inexperienced versus the two-sided alternative is $W=18, n=3$, $\mathrm{m}=34, \mathrm{p}$-value $=0.0263$; for Rekindle versus twice-experienced it is $\mathrm{W}=28, \mathrm{n}=3, \mathrm{~m}=8, \mathrm{p}$ value $=0.0485$. Result 5 shows that the interaction between volume and amplitude is lessened with the rekindle experience relative to inexperienced subjects. However, there is a lingering effect since this scalar value is higher when Rekindle is compared to twiceexperienced subjects.

Results $1-4$ cast doubts on the robustness of experience including any 'error' that is eliminated by experience. New elements in the environment, not part of the world of the experienced population, rekindle major generic characteristics of these asset market bubbles.

The rekindling of the bubble can thus be a result of two forces: (1) the impact of the 'shock' involved in changing parameters between second and third experience, which induces a need for people to adapt to the new environment, and/or (2) the sheer force of 
the values of the parameters chosen for the rekindle treatment, which may induce bubbles regardless of experience. At first glance, Results 1- 4 imply the former. To test for the latter, we look at the outcome of the new replication treatments.

Result 5: Experience fails to reduce amplitude under the new parameters. There is no significant difference between the amplitude of the bubble in the inexperienced baseline environment and that of the bubbles in inexperienced or twice experienced under the new replication environment.

The Exact Wilcoxon Rank sum test for the null hypothesis that the InexperiencedNewRep amplitude is the same as inexperienced versus the two-sided alternative is $\mathrm{W}=78, \mathrm{n}=3, \mathrm{~m}=34$, $\mathrm{p}$-value $=0.2680$; for Twice-experiencedNewRep versus inexperienced it is $\mathrm{W}=38, \mathrm{n}=3, \mathrm{~m}=34, \mathrm{p}$-value $=0.3189$. Result 4 shows that the environment of the new replication sessions is robust to three experiences. High liquidity and dividend spread contribute to maintaining a substantial bubble despite subjects' increased familiarity with the environment. Unlike the baseline, learning fails to transfer under new replication, and the bubble maintains its height.

Result 6: Experience significantly reduces the duration of a bubble. Duration falls just as dramatically and significantly under the new replication environment as under the baseline environments. It is also reduced relative to the response in Rekindle. 
The Exact Wilcoxon Rank sum test for the null hypothesis that the TwiceexperiencedNewRep duration is the same as twice-experienced versus the two-sided alternative is $\mathrm{W}=18, \mathrm{n}=3, \mathrm{~m}=8, \mathrm{p}$-value $=1.0000$; for Twice-experiencedNewRep versus rekindle it is $\mathrm{W}=6.5, \mathrm{n}=3, \mathrm{~m}=3, \mathrm{p}$-value $=0.2000$. Just as in result 2 , result 6 shows that a residual effect of experience allows learning to transfer for duration despite the high liquidity, high dividend spread environment. Thus, bubbles do not last as long as traders gain more experience. However, there appears to be a greater transfer of learning under a static environment (as in New Replication) as opposed to an altered environment (as in Rekindle).

Result 7: Experience significantly reduces turnover. Turnover in Twice-experienced-New Replication is significantly less than turnover with inexperienced traders.

The Exact Wilcoxon Rank sum test for the null hypothesis that the TwiceexperiencedNewRep turnover is the same as the inexperienced baseline is $W=12, n=3$, $\mathrm{m}=34, \mathrm{p}=$ value $=0.0059$. Result 7 suggests that experience is robust to a high liquidity, high dividend static environment in reducing turnover. Subjects learn through experience to trade less despite any increased temptation to spend their [higher] cash endowment.

Result 8: Market value amplitude is reduced with experience under the new parameters. The Exact Wilcoxon Rank sum test for the null hypothesis that the TwiceexperiencedNewRep market value amplitude is the same as the twice-experienced baseline is $\mathrm{W}=25, \mathrm{n}=3, \mathrm{~m}=8, \mathrm{p}=\mathrm{value}=0.1939$. Consistent with result 4 , result 8 shows 
that the interaction between volume and amplitude is lessened over experience regardless of environment.

\section{Conclusion}

This study has focused on the robustness of learning and 'error' elimination on participants in a laboratory asset market and its effect on price bubbles. The results show that experience has a strong effect in a particular, commonly used stationary environment. Moreover, the results of DLM demonstrate that there exist environments in which experience can stifle the exuberance of inexperienced traders and squelch a bubble. Our results, however, while using the standard SSW environment, suggest that experience alone is not a sufficient condition to ensure the elimination of price bubbles. In particular, when important elements in the underlying market environment change for experienced subjects, a bubble can reignite. But our control experiments establish that if the environment is one of high liquidity and high dividend spread, a bubble can be sustained in amplitude despite experience. Therefore, our shock effort to reignite bubbles with twice-experienced subjects is successful only in respect to the duration of a bubble; the high amplitude which reappears is more a function of the environment than the shock itself. As a byproduct of this effort we offer an enlarged database consisting of 81 experiments across three levels of subject experience with three different variations on the economic environment.

Experience, including possible 'error' elimination, is robust only to a very particular environment in determining the characteristics of a price bubble. Since experience 
changes with the turnover of investors, but also the underlying environment changes in national stock markets, we offer these new experiments as relevant for interpreting the relationship between laboratory and field asset market observations. 


\section{References}

Ackert, Lucy, Narat Charupat, Bryan Church, and Richard Deaves. 2006. "Margin, Short Selling, and Lotteries in Experimental Asset Markets." Southern Economic Journal, 73(2): 419-436.

Caginalp, Gunduz, David Porter, and Vernon Smith. 1998. "Initial Cash/Stock Ratio and Stock Prices: An Experimental Study.” Proceedings of the National Academy of Sciences, 95(2): 756-761.

Caginalp, Gunduz, David Porter, and Vernon Smith. 2000. "Momentum and Overreaction in Experimental Asset Markets." International Journal of Industrial Organization, 18(1): 187-204.

Caginalp, Gunduz, David Porter, and Vernon Smith. 2001. "Financial Bubbles: Excess Cash, Momentum, and Incomplete Information." Journal of Behavioral Finance, 2(2): $80-99$.

Dufwenberg, Martin, Tobias Lindqvist, and Evan Moore. 2005. "Bubbles and Experience: An Experiment.” American Economic Review, 95(5): 1731-1737.

Haruvy, Ernan and Charles Noussair. 2006. "The Effect of Short Selling on Bubbles and Crashes in Experimental Spot Asset Markets." Journal of Finance, 61(3): 1119-1157. 
Haruvy, Ernan, Yaron Lahav, and Charles Noussair. Forthcoming. "Traders'

Expectations in Asset Markets: Experimental Evidence.” American Economic Review.

King, Ronald, Vernon Smith, Arlington Williams, and Mark Van Boening. 1993. "The Robustness of Bubbles and Crashes in Experimental Stock Markets.” In Nonlinear Dynamics and Evolutionary Economics, ed. Richard Day and Ping Chen, 183-200. Oxford: Oxford University Press.

Lei, Vivian, Charles Noussair, and Charles Plott. 2001. "Nonspeculative Bubbles in Experimental Asset Markets: Lack of Common Knowledge of Rationality vs. Actual Irrationality." Econometrica, 69(4): 831-859.

McCabe, Kevin and Vernon Smith. 2000. "A Comparison of Naïve and Sophisticated Subject Behavior with Game Theoretic Predictions." Proceedings of the National Academy of Sciences, 97(7): 3777-3781.

Noussair, Charles and Steven Tucker. 2006. "Futures Markets and Bubble Formation in Experimental Asset Markets.” Pacific Economic Review, 11(2): 167-184.

Peterson, Steven. 1993. "Forecasting Dynamics and Convergence to Market Fundamentals: Evidence from Experimental Asset Markets." Journal of Economic Behavior and Organization, 22(3): 269-284. 
Porter, David and Vernon Smith. 1995. "Futures Contracting and Dividend Uncertainty in Experimental Asset Markets." Journal of Business, 68(4): 509-541.

Smith, Vernon, Gerry Suchanek, and Arlington Williams. 1988. "Bubbles, Crashes, and Endogenous Expectations in Experimental Spot Asset Markets." Econometrica, 56(5): $1119-1151$.

Smith, Vernon. 2003. "Constructivist and Ecological Rationality in Economics." The American Economic Review, 93(3): 465-508.

Van Boening, Mark, Arlington Williams, and Shawn LaMaster. 1993. "Price Bubbles and Crashes in Experimental Call Markets." Economics Letters, 41(2): 179-185. 
$\underline{\text { Tables }}$

Table 1: Baseline Treatment Initial Trader Portfolios and Expected Values Each experiment had three trader types. Subjects were evenly distributed across the trader types with any remainders being assigned to the medium portfolio.

\begin{tabular}{cccc} 
Trader Type & Initial Cash & Initial Shares & $\begin{array}{c}\text { Expected } \\
\text { Value }\end{array}$ \\
\hline \hline High Cash / Low Shares & $\$ 10.80$ & 3 & $\$ 21.60$ \\
Medium Cash / Medium Shares & $\$ 7.20$ & 4 & $\$ 21.60$ \\
Low Cash / High Shares & $\$ 3.60$ & 5 & $\$ 21.60$
\end{tabular}




\section{Table 2: Rekindle Treatment Initial Trader Portfolios and Expected Values}

Each experiment had three trader types. Subjects were evenly distributed across the trader types with any remainders being assigned to the medium portfolio. The portfolios were reduced by 2 -shares for each type relative to the baseline treatment with the expected dividend value of the 2-shares (810) added to the initial cash position.

\begin{tabular}{cccc} 
Trader Type & $\begin{array}{c}\text { Initial } \\
\text { Cash }\end{array}$ & $\begin{array}{c}\text { Initial } \\
\text { Shares }\end{array}$ & $\begin{array}{c}\text { Expected } \\
\text { Value }\end{array}$ \\
\hline \hline High Cash / Low Shares & $\$ 18.90$ & 1 & $\$ 22.95$ \\
Medium Cash / Medium Shares & $\$ 15.30$ & 2 & $\$ 23.40$ \\
Low Cash / High Shares & $\$ 11.70$ & 3 & $\$ 23.85$
\end{tabular}


Table 3: Description of Samples to Be Used in the Regressions (Table 4)
Along with the data from our database, we report 10 replications of experiments under baseline parameters, 3 with the rekindle environment parameters using (mixed) subjects from these replications, and 9 New Replication experiments using the same rekindle environment parameters across all three experience levels (not mixed).

\begin{tabular}{cccccccc}
$\begin{array}{c}\text { Experience } \\
\text { Level }\end{array}$ & Database & Replications & $\begin{array}{c}\text { Replications } \\
\text { for Rekindle } \\
\text { (Baseline } \\
\text { Parameter) }\end{array}$ & Subtotal & $\begin{array}{c}\text { Rekindle } \\
\text { Treatment }\end{array}$ & $\begin{array}{c}\text { New } \\
\text { Replications }\end{array}$ & $\begin{array}{c}\text { Total } \\
\text { Experiments }\end{array}$ \\
\hline $\begin{array}{c}\text { Inexperienced } \\
\begin{array}{c}\text { Once- } \\
\text { Experienced }\end{array}\end{array}$ & 34 & 2 & 5 & 41 & NA & 3 & 44 \\
$\begin{array}{c}\text { Twice- } \\
\text { Experienced }\end{array}$ & 13 & 2 & 5 & 20 & NA & 3 & 23 \\
Total & 53 & 6 & NA & 8 & $3(\text { mixed) })^{1}$ & 3 & 14 \\
& 5 & 10 & 69 & 3 & 9 & 81
\end{tabular}

NA Not applicable

${ }^{1}$ Subjects are drawn from the pool of 5 experienced, then mixed; in all others subjects are kept in the same groups across experience levels. 
Table 4: SUR estimates of Amplitude, Duration and Turnover

The independent variables in each numbered column are dummy variables of the treatments. The estimates are marginal

effects measured relative to the inexperienced 'mean' (the intercept estimate). The t-statistic along with the number of observations for the treatment is provided below the estimate.

\begin{tabular}{|c|c|c|c|c|c|c|c|c|c|c|}
\hline & $\begin{array}{c}\text { (Intercept) } \\
\text { Inexperienced }\end{array}$ & $\begin{array}{c}\text { Once- } \\
\text { experienced }\end{array}$ & $\begin{array}{c}\text { Twice- } \\
\text { experienced }\end{array}$ & Rekindle & $\begin{array}{l}\text { Inexperienced } \\
\text { New Replication }\end{array}$ & $\begin{array}{l}\text { Once-experienced } \\
\text { New Replication }\end{array}$ & $\begin{array}{c}\text { Twice- } \\
\text { experienced New } \\
\text { Replication }\end{array}$ & $\begin{array}{l}\text { Inexperience } \\
\text { Replications }\end{array}$ & $\begin{array}{c}\text { Once- } \\
\text { Experienced } \\
\text { Replications }\end{array}$ & $\mathbf{R} 2$ \\
\hline \multirow{3}{*}{ Amplitude } & 1.2373 & -0.4295 & -1.008 & -0.0949 & 0.2359 & 0.0791 & -0.2069 & 0.0779 & -0.3416 & \multirow{3}{*}{0.45} \\
\hline & $\mathrm{t}=18.6761$ & $\mathrm{t}=-3.5202$ & $t=-6.5777$ & $\mathrm{t}=-0.4037$ & $\mathrm{t}=1.0032$ & $\mathrm{t}=0.3365$ & $\mathrm{t}=-0.8796$ & $\mathrm{t}=0.4814$ & $\mathrm{t}=-1.9694$ & \\
\hline & $\mathrm{N}=34$ & $\mathrm{~N}=13$ & $\mathrm{~N}=8$ & $\mathrm{~N}=3$ & $\mathrm{~N}=3$ & $\mathrm{~N}=3$ & $\mathrm{~N}=3$ & $\mathrm{~N}=7$ & $\mathrm{~N}=7$ & \\
\hline \multirow{3}{*}{ Duration } & 9.2299 & -2.7509 & -6.854 & -4.5623 & -1.5623 & -4.229 & -6.8956 & 1.1849 & -2.6723 & \multirow{3}{*}{0.56} \\
\hline & $t=21.6298$ & $\mathrm{t}=-3.5007$ & $t=-6.9445$ & $\mathrm{t}=-3.0121$ & $\mathrm{t}=-1.0315$ & $\mathrm{t}=-2.7921$ & $\mathrm{t}=-4.9928$ & $\mathrm{t}=1.1930$ & $\mathrm{t}=-2.6904$ & \\
\hline & $\mathrm{N}=34$ & $\mathrm{~N}=13$ & $\mathrm{~N}=8$ & $\mathrm{~N}=3$ & $\mathrm{~N}=3$ & $\mathrm{~N}=3$ & $\mathrm{~N}=3$ & $\mathrm{~N}=7$ & $\mathrm{~N}=7$ & \\
\hline \multirow{3}{*}{ Turnover } & 3.074 & -0.2071 & -1.8568 & -0.9793 & -0.4295 & -1.0406 & -1.5629 & -0.3255 & -1.3269 & \multirow{3}{*}{0.37} \\
\hline & $t=19.6487$ & $\mathrm{t}=-0.7189$ & $\mathrm{t}=-5.1309$ & $t=-1.7633$ & $\mathrm{t}=-0.7734$ & $\mathrm{t}=-1.8738$ & $\mathrm{t}=-2.8141$ & $\mathrm{t}=-0.9212$ & $\mathrm{t}=-3.2394$ & \\
\hline & $\mathrm{N}=34$ & $\mathrm{~N}=13$ & $\mathrm{~N}=8$ & $\mathrm{~N}=3$ & $\mathrm{~N}=3$ & $\mathrm{~N}=3$ & $\mathrm{~N}=3$ & $\mathrm{~N}=7$ & $\mathrm{~N}=7$ & \\
\hline \multirow{3}{*}{ Market Value } & 5.4296 & -2.49 & -4.7691 & -3.5165 & -2.9323 & -4.0199 & -4.0169 & -0.4512 & -2.6883 & \multirow{3}{*}{0.23} \\
\hline & $\mathrm{t}=9.0572$ & $\mathrm{t}=-2.2554$ & $\mathrm{t}=-3.493$ & $t=-1.6525$ & $\mathrm{t}=-1.3779$ & $\mathrm{t}=-1.8890$ & $\mathrm{t}=-1.8876$ & $t=-0.3080$ & $\mathrm{t}=-1.7127$ & \\
\hline & $\mathrm{N}=34$ & $\mathrm{~N}=17$ & $\mathrm{~N}=8$ & $\mathrm{~N}=3$ & $\mathrm{~N}=3$ & $\mathrm{~N}=3$ & $\mathrm{~N}=3$ & $\mathrm{~N}=3$ & $\mathrm{~N}=3$ & \\
\hline
\end{tabular}




\section{Table 5: Paired Comparisons using Exact Wilcoxon Rank Sum Statistic}

For each of the treatments the Wilcoxon W-statistic is provided along with the sample sizes for each treatment and the p-value.

\begin{tabular}{|c|c|c|c|c|c|c|c|c|c|c|c|c|}
\hline & \multicolumn{3}{|c|}{ Amplitude } & \multicolumn{3}{|c|}{ Duration } & \multicolumn{3}{|c|}{ Turnover } & \multicolumn{3}{|c|}{ Market Value } \\
\hline & Rekindle & $\begin{array}{c}\text { New } \\
\text { Replication } \\
\text { Inexperienc } \\
\text { ed }\end{array}$ & $\begin{array}{c}\text { New } \\
\text { Replication } \\
\text { Twice- } \\
\text { Experienced }\end{array}$ & Rekindle & $\begin{array}{c}\text { New } \\
\text { Replication } \\
\text { Inexperienced }\end{array}$ & $\begin{array}{c}\text { New } \\
\text { Replication } \\
\text { Twice- } \\
\text { Experienced }\end{array}$ & Rekindle & $\begin{array}{c}\text { New } \\
\text { Replication } \\
\text { Inexperience } \\
\mathrm{d}\end{array}$ & $\begin{array}{c}\text { New } \\
\text { Replication } \\
\text { Twice- } \\
\text { Experienced }\end{array}$ & Rekindle & $\begin{array}{c}\text { New } \\
\text { Replication } \\
\text { Inexperienced }\end{array}$ & $\begin{array}{c}\text { New } \\
\text { Replication } \\
\text { Twice- } \\
\text { Experienced }\end{array}$ \\
\hline \multirow{4}{*}{$\begin{array}{l}\text { Baseline } \\
\text { Inexp }\end{array}$} & $\mathrm{W}=49$ & $\mathrm{~W}=78$ & $\mathrm{~W}=38$ & $\mathrm{~W}=11$ & $\mathrm{~W}=32$ & $\mathrm{~W}=6$ & $\mathrm{~W}=31$ & $\mathrm{~W}=53.5$ & $\mathrm{~W}=12$ & $\mathrm{~W}=18$ & $\mathrm{~W}=23$ & $\mathrm{~W}=15$ \\
\hline & $n=3$ & $\mathrm{n}=3$ & $\mathrm{n}=3$ & $\mathrm{n}=3$ & $\mathrm{n}=3$ & $n=3$ & $\mathrm{n}=3$ & $\mathrm{n}=3$ & $\mathrm{n}=3$ & $\mathrm{n}=3$ & $\mathrm{n}=3$ & $\mathrm{n}=3$ \\
\hline & $\mathrm{m}=34$ & $\mathrm{~m}=34$ & $\mathrm{~m}=34$ & $\mathrm{~m}=34$ & $\mathrm{~m}=34$ & $\mathrm{~m}=34$ & $\mathrm{~m}=34$ & $\mathrm{~m}=34$ & $\mathrm{~m}=34$ & $\mathrm{~m}=34$ & $\mathrm{~m}=34$ & $\mathrm{~m}=34$ \\
\hline & $\mathrm{p}=0.6932$ & $\mathrm{p}=0.2680$ & $\mathrm{p}=0.3189$ & $\mathrm{p}=0.0041$ & $\mathrm{p}=0.1701$ & $\mathrm{p}=0.0000$ & $\mathrm{p}=0.1647$ & $\mathrm{p}=0.8584$ & $\mathrm{p}=0.0059$ & $\begin{array}{c}\mathrm{p}=0.026 \\
3\end{array}$ & $\mathrm{p}=0.0610$ & $\mathrm{p}=0.0136$ \\
\hline \multirow{4}{*}{$\begin{array}{c}\text { Baseline } \\
\text { Twice-exp }\end{array}$} & $\mathrm{W}=30$ & $\mathrm{~W}=30$ & $\mathrm{~W}=30$ & $\mathrm{~W}=27$ & $\mathrm{~W}=30$ & $\mathrm{~W}=18$ & $\mathrm{~W}=27$ & $\mathrm{~W}=30$ & $\mathrm{~W}=25.5$ & $\mathrm{~W}=28$ & $\mathrm{~W}=28$ & $\mathrm{~W}=25$ \\
\hline & $\mathrm{n}=3$ & $\mathrm{n}=3$ & $\mathrm{n}=3$ & $\mathrm{n}=3$ & $\mathrm{n}=3$ & $\mathrm{n}=3$ & $\mathrm{n}=3$ & $\mathrm{n}=3$ & $\mathrm{n}=3$ & $\mathrm{n}=3$ & $\mathrm{n}=3$ & $\mathrm{n}=3$ \\
\hline & $\mathrm{m}=8$ & $\mathrm{~m}=8$ & $\mathrm{~m}=8$ & $\mathrm{~m}=8$ & $\mathrm{~m}=8$ & $\mathrm{~m}=8$ & $\mathrm{~m}=8$ & $\mathrm{~m}=8$ & $\mathrm{~m}=8$ & $\mathrm{~m}=8$ & $\mathrm{~m}=8$ & $\mathrm{~m}=8$ \\
\hline & $\mathrm{p}=0.0121$ & $\mathrm{p}=0.0121$ & $\mathrm{p}=0.0121$ & $\mathrm{p}=0.0848$ & $\mathrm{p}=0.0121$ & $\mathrm{p}=1.0000$ & $\mathrm{p}=0.0848$ & $\mathrm{p}=0.0121$ & $\mathrm{p}=0.1697$ & $\begin{array}{c}\mathrm{p}=0.048 \\
5\end{array}$ & $\mathrm{p}=0.0485$ & $\mathrm{p}=0.1939$ \\
\hline \multirow{4}{*}{ Rekindle } & \multirow{4}{*}{ NA } & $\mathrm{W}=8$ & $\mathrm{~W}=9$ & \multirow{4}{*}{ NA } & $\mathrm{W}=6$ & $\mathrm{~W}=6.5$ & \multirow{4}{*}{ NA } & $\mathrm{W}=8$ & $\mathrm{~W}=9$ & \multirow{4}{*}{ NA } & $\mathrm{W}=10$ & $\mathrm{~W}=9$ \\
\hline & & $\mathrm{n}=3$ & $\mathrm{n}=3$ & & $\mathrm{n}=3$ & $\mathrm{n}=3$ & & $\mathrm{n}=3$ & $\mathrm{n}=3$ & & $\mathrm{n}=3$ & $\mathrm{n}=3$ \\
\hline & & $\mathrm{m}=3$ & $\mathrm{~m}=3$ & & $\mathrm{~m}=3$ & $\mathrm{~m}=3$ & & $\mathrm{~m}=3$ & $\mathrm{~m}=3$ & & $\mathrm{~m}=3$ & $\mathrm{~m}=3$ \\
\hline & & $\mathrm{p}=0.4$ & $\mathrm{p}=0.7$ & & $\mathrm{p}=0.1000$ & $\mathrm{p}=0.2000$ & & $\mathrm{p}=0.4000$ & $\mathrm{p}=0.7000$ & & $\mathrm{p}=1.0000$ & $\mathrm{p}=0.7000$ \\
\hline
\end{tabular}




\section{$\underline{\text { Figures }}$}

Figure 1: Average Price Deviation from Fundamental Value for Various Experience Levels [from Dufenberg et al. (2005)]

The graph charts the difference between market price and fundamental value and each period for inexperienced, once-experience and twice-experienced treatments of the Dufwenberg et al. (2005) experiments.

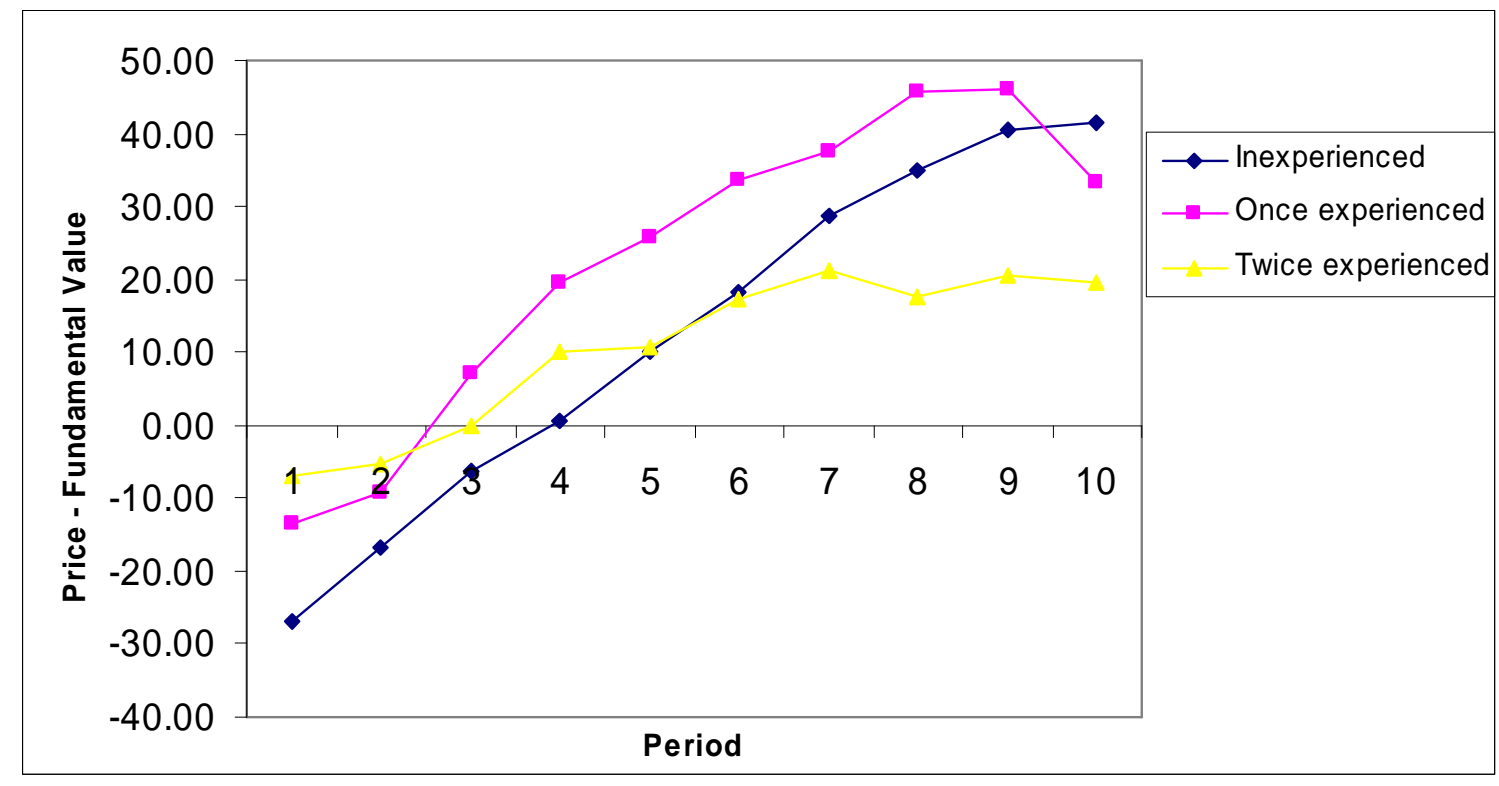


Figure 2: Prices and Trade Volume for Various Levels of Cohort Experience The graph charts differences between price and fundamental value each period for the same cohort of subjects that participated in three sessions (inexperienced, onceexperienced and twice-experienced) of an asset market. The trade volume each period is given by the number next to the contract price symbol. ${ }^{11}$

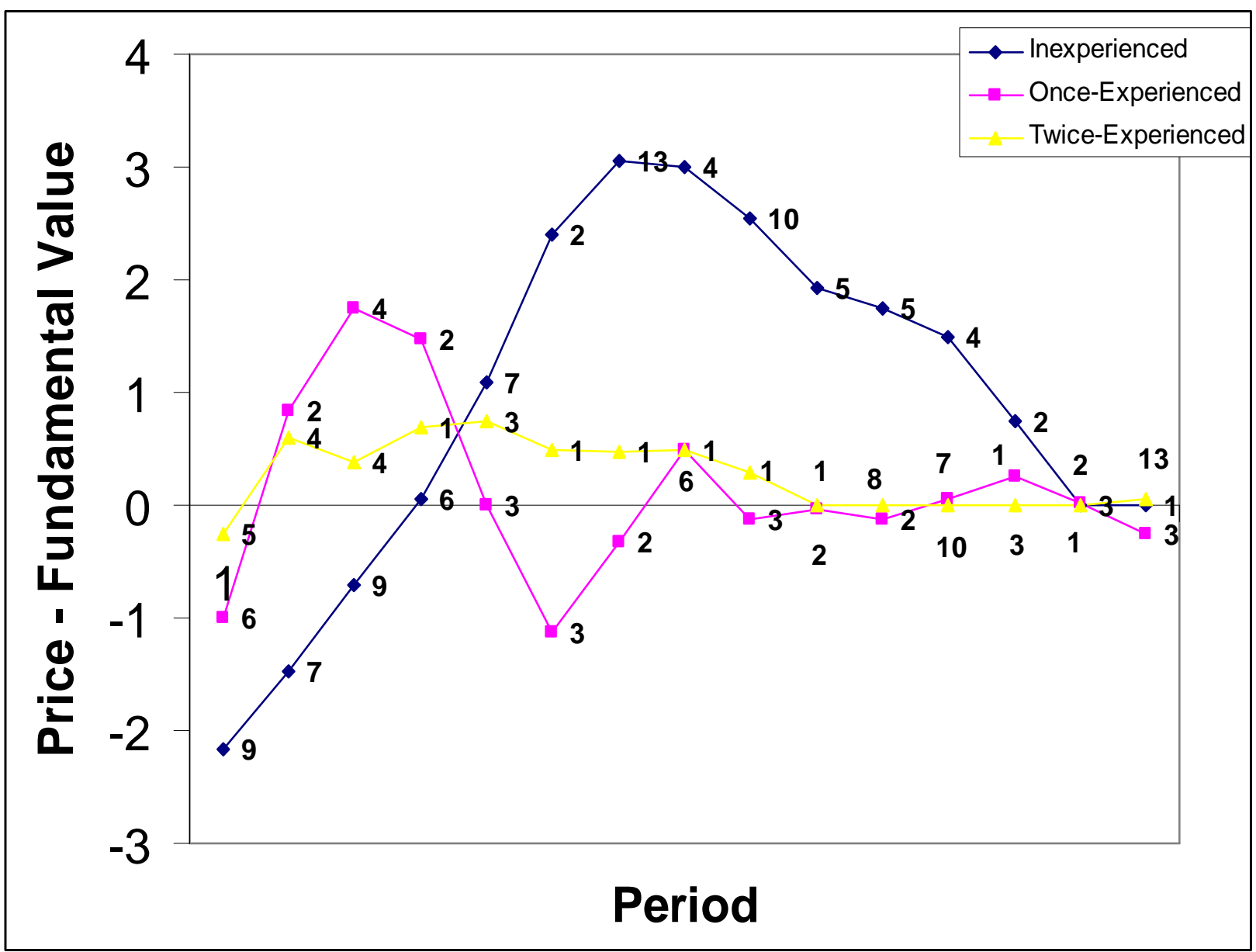


Figure 3: Replication Experiment Results

The graph charts the difference between fundamental value and the market price each period for inexperienced, once-experience and twice-experienced treatments of the replication experiments.

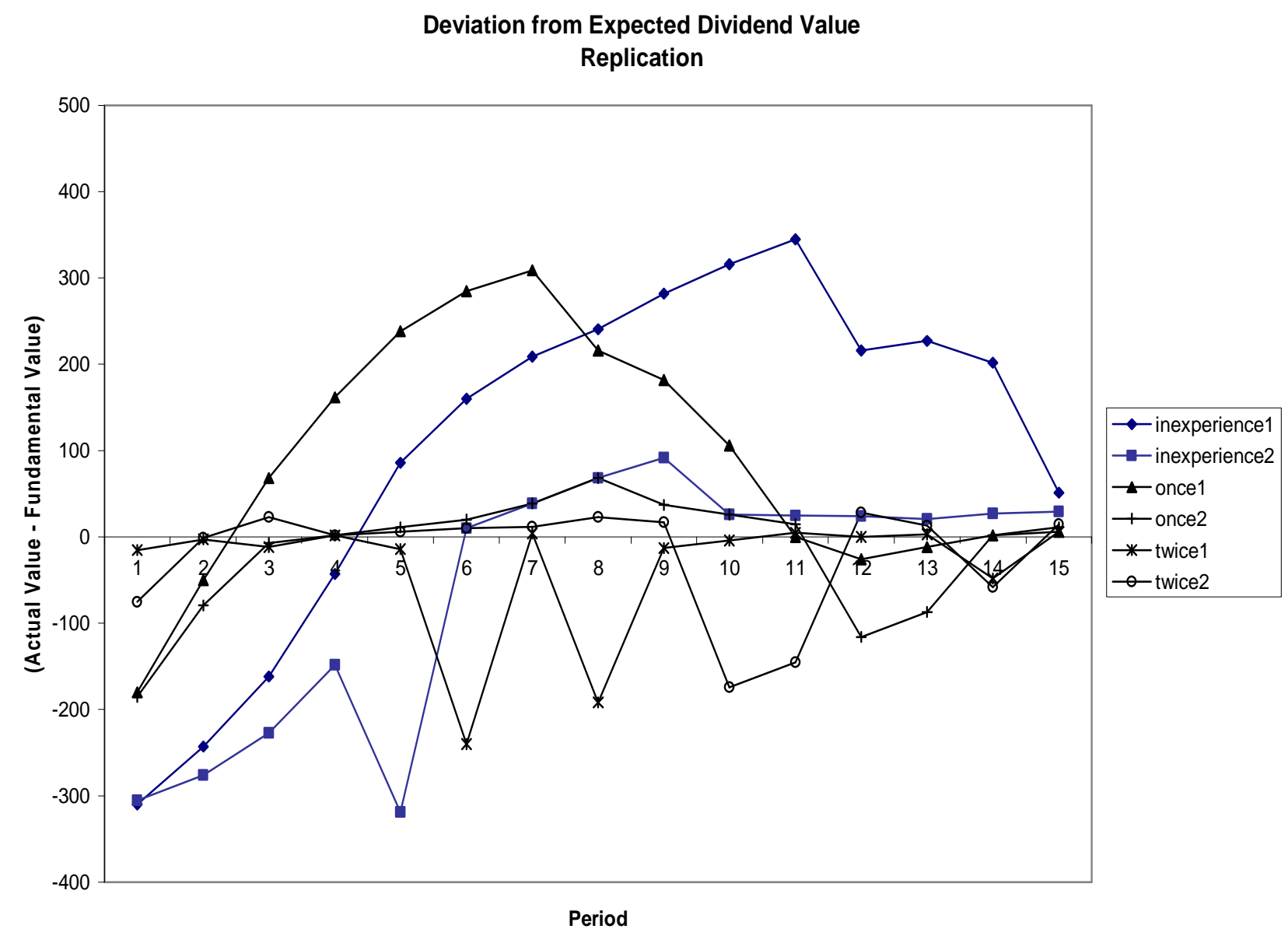


Figure 4: Time Series Price Deviation from Fundamental Value for the Rekindle and Twice-Experienced Baseline Replication

The graph charts the difference between fundamental value and the market price each period of the twice-experienced subjects in the replication and rekindle experiments.

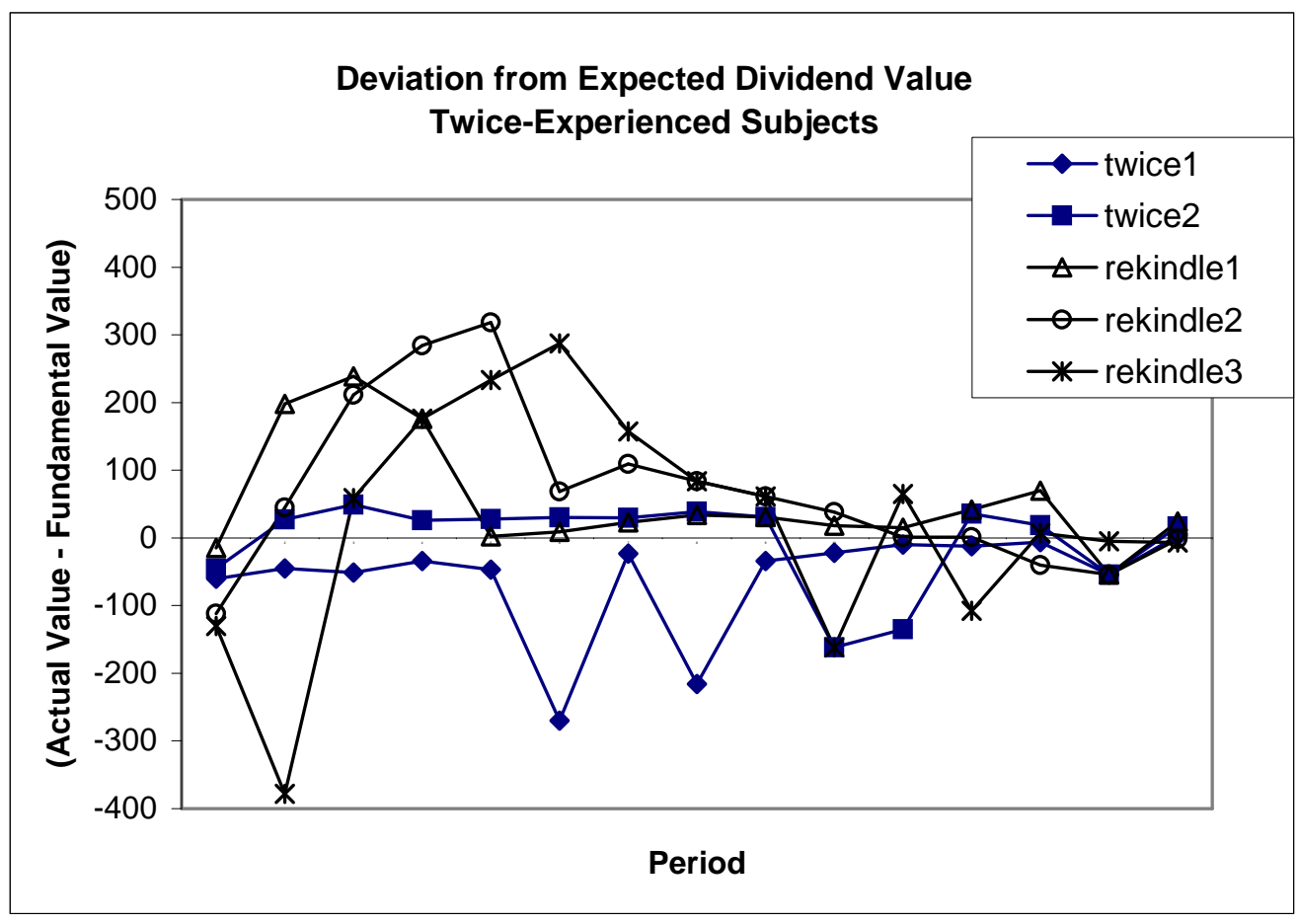


Figure 5: Time Series Price Deviation from Fundamental Value for the TwiceExperienced New Replication Sessions and the Twice-Experienced Baseline

\section{Replication}

The graph charts the difference between fundamental value and the market price each period of the twice-experienced subjects in the replication and new replication experiments.

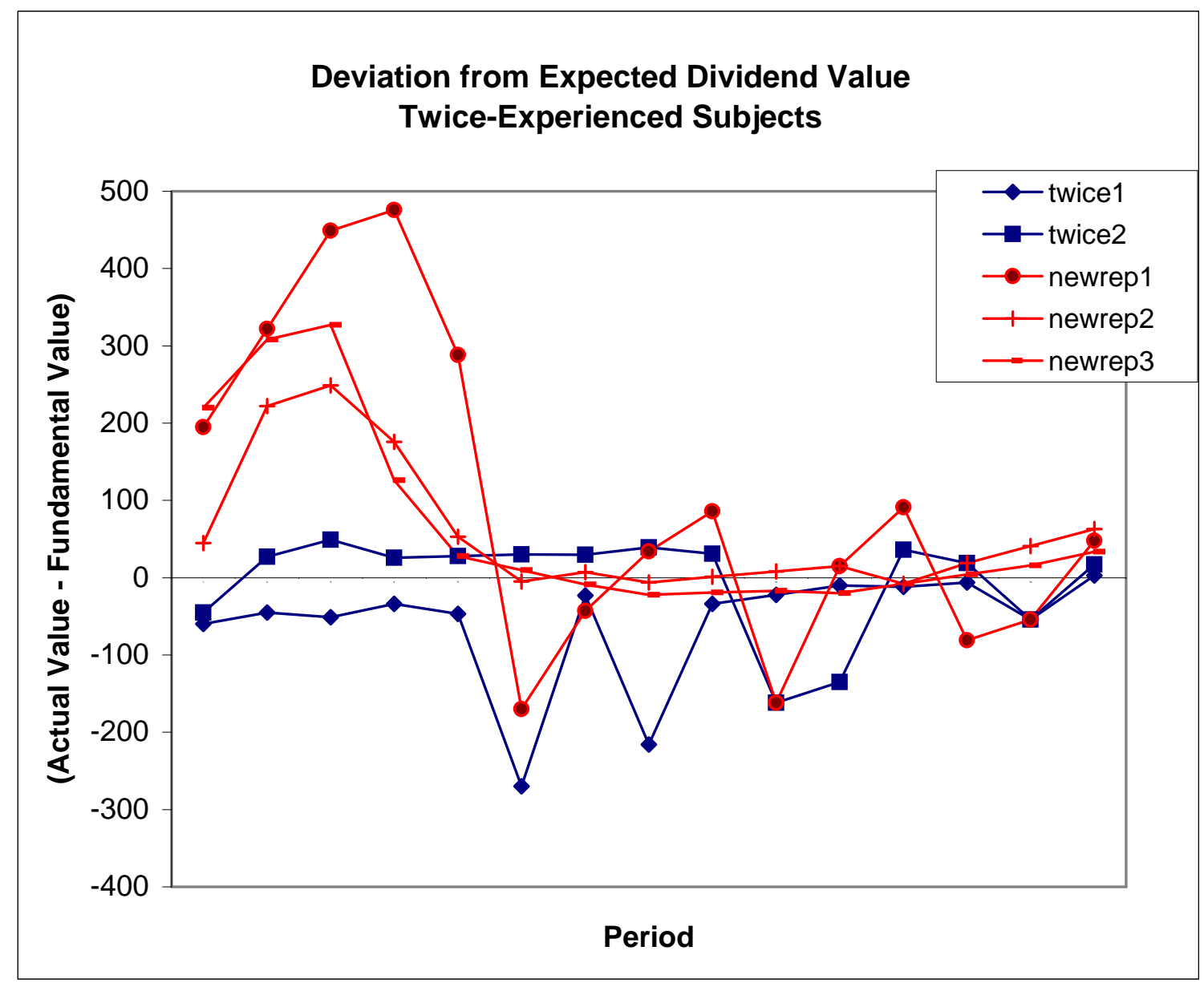

